\title{
RESERVOIR OFFSET MODELS FOR RADIOCARBON CALIBRATION
}

\author{
Martin Jones \\ Centre for Archaeological Research, University of Auckland, Private Bag 92019, Auckland, New Zealand. \\ Email: Martin@analytic.co.nz. \\ Geoff Nicholls \\ Department of Mathematics, University of Auckland, Private Bag 92019, Auckland, New Zealand. \\ Email: Nicholls@math.auckland.ac.nz.
}

\begin{abstract}
The purpose of a reservoir offset is to enable the application of calibration data $(\mu(\theta)$, e.g. Stuiver et al. 1998) developed for one reservoir (primary reservoir) to CRAs from another (secondary reservoir), for example the use of a hemispheric offset for terrestrial samples (Barbetti et al. 1995; McCormac et al. 1998; Sparks et al. 1995; Vogel et al. 1986, 1993). The usual approach has been to define the activity of the secondary reservoir as some form of constant offset (with error) from the primary reservoir (e.g. Higham and Hogg 1985; McFadgen and Manning 1990). In this case, all CRAs from a secondary reservoir are given the same offset. The value of this common offset is not known exactly, but any uncertainty in the measured value of the offset corresponds to uncertainty in the common offset for all CRAs. However, the standard procedure for incorporating offset error into CRAs incorrectly allows a different offset for each CRA. The offset for each CRA is incorrectly allowed to vary by the measurement error reported for the offset value. Technically, the offset is incorrectly treated as varying independently from one CRA to the next, when in fact it is a single parameter for the secondary reservoir in question. In light of this, the calibrated date distributions will be incorrect for CRAs where an offset has been applied and the standard approach to offset error treatment has been used. In many cases, the differences between correct and incorrect calibrated date distributions will be insignificant. However, in some cases significant differences may arise and other approaches to treating the error associated with offsets need to be adopted.
\end{abstract}

\section{INTRODUCTION}

Reservoir offsets are commonly applied in the calibration of conventional radiocarbon ages (CRAs) deriving from reservoirs for which primary calibration data are unavailable. For example, offsets are sometimes used in the calibration of terrestrial samples in the southern hemisphere (Barbetti et al. 1995; McCormac et al. 1998; Sparks et al. 1995; Vogel et al. 1986, 1993). Here the terrestrial calibration data are defined for a northern hemisphere atmospheric reservoir (the primary reservoir in this case) and an offset may be needed to enable these data to be applied to samples from a southern hemisphere atmospheric reservoir (the secondary reservoir).

Regardless of what functional form the offset takes, it will have an associated uncertainty. The contribution of this uncertainty to the final uncertainty in the calibrated date must be taken into account when that CRA is calibrated. The standard approach is to incorporate offset uncertainty into the CRA error prior to calibration as follows. Suppose $K$ CRAs $\left(y_{i}, i=1,2 \ldots K\right)$ are measured, with corresponding standard errors $\left(\sigma_{i}, i=1,2 \ldots K\right)$ and the offset for the reservoir in question has been measured to be $\delta$ with standard deviation $\sigma_{\delta}$. Standard practice is to combine the error in the CRA with the error in the offset to produce a single number $\sigma_{i}^{\prime}$ taking into account the two sources of error, i.e.

$$
\sigma_{i}^{\prime}=\sqrt{\sigma_{i}^{2}+\sigma_{\delta}^{2}}
$$

Generating a new error, $\sigma_{i}^{\prime}$, for each CRA which is then calibrated as normal. However, this is technically incorrect. The problem is that the formula above treats the offset as a quantity which varies independently from one CRA to the next. This is essentially the same as asserting that each CRA analyzed derives from a different secondary reservoir. Thus if we wish to analyze a number of CRAs from the same secondary reservoir (for example southern hemisphere terrestrial samples) we cannot incorporate the offset error into the CRA following Equation (1). This means that we must take account of the offset uncertainty in the calibration process via a different approach. To correctly 
incorporate reservoir offset error into calibrated date distributions, it is necessary to identify an appropriate observation model.

\section{Observation Models}

The models defined here follow the standard form in widespread use, e.g. Buck et al. 1996; Nicholls and Jones 1998. Let $\theta_{i}$ denote the calibrated age parameter associated with the $i$ th CRA. Given that a constant offset is used, the correct observation model (model 1) for CRAs $\left(y_{i}, i=1,2 \ldots K\right)$ from the $R$-reservoir is

$$
\begin{gathered}
y_{i}=\mu\left(\theta_{i}\right)+\varepsilon_{i}+\varepsilon\left(\theta_{i}\right)+R \\
\varepsilon_{i} \sim N\left(0, \sigma_{i}\right) \\
\varepsilon\left(\theta_{i}\right) \sim N\left(0, \sigma\left(\theta_{i}\right)\right) \\
R \sim N\left(\delta, \sigma_{\delta}\right) .
\end{gathered}
$$

According to this model the process mapping a calibrated date $\theta_{i}$ to a CRA $y_{i}$ is as follows: first there is the deterministic calibration $\mu(\theta)$; this is offset by $R$ to take us from the calibration reservoir to the R-reservoir; to this we add a normal random quantity $\varepsilon\left(\theta_{i}\right)$ arising from uncertainty $\sigma_{\mu}\left(\theta_{i}\right)$ in the calibration curve $\mu$; the CRA measurement yielding $y_{i}$ adds another normal random quantity $\varepsilon_{i}$. We do not know the values of the quantities $R, \varepsilon\left(\theta_{i}\right)$ and $\varepsilon_{i}$, but we do know, or can at least model, their distributions. Thus the random variation $\varepsilon\left(\theta_{i}\right)$ arising from uncertainty in the calibration curve is treated as a normal random variable with distribution $N\left(0, \sigma_{\mu}\left(\theta_{i}\right)\right)$, that is, mean zero and standard deviation $\sigma_{\mu}\left(\theta_{i}\right)$. These formulae determine an explicit formula for the likelihood of $\theta_{i}$ given the data $y_{i}$.

In this case it is not possible to algebraically combine the model error terms to generate an error for $y_{i}$ after Equation (1) as the CRAs $\left(y_{i}\right)$ are not independent, each containing the common term $R$. However, the standard approach is to combine the error terms as given in Equation (1). This calculation follows from an assumption that the CRAs are independent according to the following observation model (model 2)

$$
\begin{aligned}
y_{i}=\mu\left(\theta_{i}\right) & +\varepsilon_{i}+\varepsilon\left(\theta_{i}\right)+R_{i} \\
\varepsilon_{i} & \sim N\left(0, \sigma_{i}\right) \\
\varepsilon\left(\theta_{i}\right) & \sim N\left(0, \sigma\left(\theta_{i}\right)\right) \\
R_{i} & \sim N\left(\delta, \sigma_{\delta}\right) .
\end{aligned}
$$

Here the CRAs are independent as the reservoir offset is allowed to differ between the CRAs with each CRA having a unique and independent offset $R_{i}$. In this case, Error combination according to Equation (1) could be applied. However, this model does not apply the same offset to all CRAs from the secondary reservoir.

Thus error combination according to Equation (1) is at odds with the assumption that there is a constant reservoir offset (i.e. Equation 2). This is because under the assumption of a constant offset each CRA has the same offset $R$ and accordingly the CRAs are not independent. This violates the basis of error combination following Equation (1).

It is easy to visualize the problem. When the uncertainty in the offset is large, and the analysis is done in the usual way, with independent offsets as in model 2, each calibrated date moves independently over the range allowed by the uncertainty in the $\delta$-measurement. However, when the analysis is performed under model 1 all the calibrated dates move as a group over their range: their CRAs suffer a uniform offset, and it is the uncertainty in that common offset leads to uncertainty in the age 
of the dates as a group. In that case, model 2 leads to a greater span in the values of the calibrated dates than model 1 .

Unfortunately, under model 1, it is not straightforward to derive an analytical formula to incorporate offset measurement uncertainty into calibrated dates in a similar fashion to that given in Equation (1). This means that it is necessary to use simulation-based statistical software for this type of calculation (Nicholls and Jones 1998). However, software packages which implement simulationbased analysis are now in widespread use (e.g. BCal: Buck et al. 1999; Oxcal: Ramsey 1995; Datelab: Jones and Nicholls 1999), and may easily be modified to take account of the issue we raise here.

\section{Experiments}

In practice, the difference between the two methods is slight when the error term associated with the reservoir offset is small. However, where reservoir offset errors are large the differences can be significant. This can be demonstrated via a simple example consisting of two dates. Here we consider two dates (NZ-7755: BP, WK-2548: BP) that relate to the same reservoir and derive from the same archaeological stratum (Anderson et al. 1996). If we artificially vary the associated offset error it can be observed that the degree of correlation between the calibrated dates differs under the two models (Figure 1; for technical details on how to perform this type of simulation see Nicholls and Jones 1998). In practice this exerts most influence on statistics such as the difference in age, or span, between the two dated samples (Figure 2). Under a constant offset model (model 1, Equation 2) the mean difference in age and distribution of span for the two samples is largely independent of the offset error. However, under an independent offset observation model (model 2, Equation 3) the mean difference in age and span is more strongly correlated with the size of the offset error.

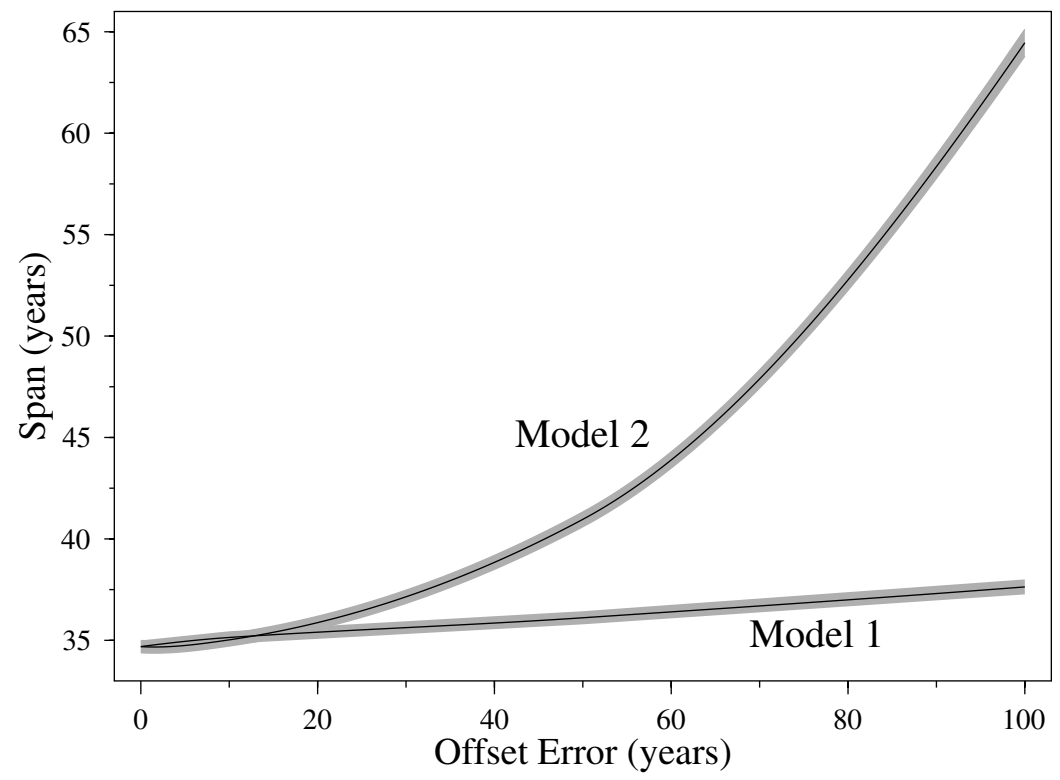

Figure 1 Span as a function of offset error under model 1 and model 2 

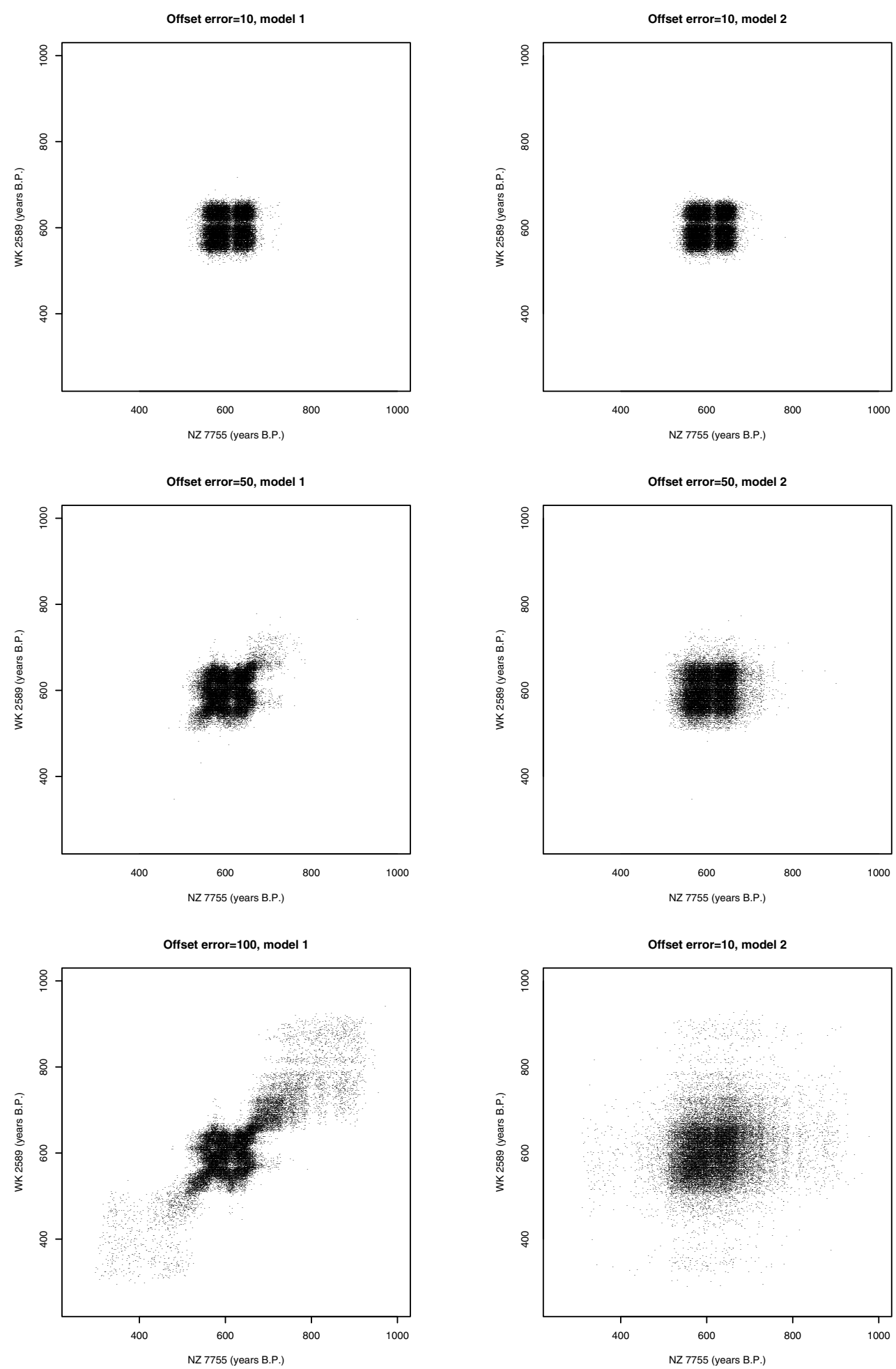

Figure 2 Correlation plots of NZ 7755 vs. WK 2546 
Obviously the degree to which critical differences in statistics and calibrated distributions occur between constant and independent offset models varies depending upon the problem under consideration. While the magnitude of error at which a significant effect occurs in the example presented are not common, more extreme examples can be found. The most significant routine reservoir offset calculation errors are likely to occur with marine samples where reservoir offsets with larger errors occur (Stuiver and Braziunas 1993). It should also be noted that this has implications for the calculation of marine calibration data derived from terrestrial data.

\section{CONCLUSION}

In the case that it is assumed that each CRA analyzed derives from an independent secondary reservoir (i.e. Equation 3), the incorporation of offset error into calibration calculations using Equation (1) is appropriate. However, other approaches should be adopted if a constant or other nonindependent reservoir offset is applied (e.g. Equation 2). The use of a constant offset that renders the observed CRAs non-independent can be calculated via approaches such as sampled based Bayesian inference where the appropriate observation model is applied (Nicholls and Jones 1998).

Most available calibration software packages only implement reservoir offsets according to the observation model given in Equation (3), and accordingly may not be appropriate for calibration where a constant reservoir offset is assumed. It is currently possible to calibrate CRAs according to the observation models given in both Equations (2) and (3) with the Datelab analysis package (Jones and Nicholls 1999). Further technical details may be found in Nicholls and Jones (1998).

Other calibrated likelihood models can be developed to incorporate factors such as temporal variance in the reservoir offset. However, all models other than the one assuming independence require analysis via a method such as sample based Bayesian inference (Buck et al. 1996; Nicholls and Jones 1998).

A similar issue to the one discussed here appears in the treatment of the standard errors in the calibration curve itself. The problem is that the calibration data are correlated, and consequently errors in the calibration curve are not strictly independent. This is considered in (Christen and Nicholls 2000) where it is shown that the effect will be unimportant except possibly for high precision measurements in which several dates have very nearly equal calibrated values.

\section{ACKNOWLEDGMENTS}

This work was supported by the FoRST under contract UOA-807.

\section{REFERENCES}

Anderson AJ, Smith IWG, Higham TFG. 1996. Radiocarbon chronology. In: Anderson AJ, Allingham B, Smith IWG, editors, Shag River Mouth: the archaeology of an early Southern Maori village. Volume 27. Canberra, Australia: ANH Publications, RSPAS, ANU. p 60-9.

Barbetti M, Bird T, Dolezal G, Taylor G, Francey R, Cook E, Petersen M. 1995. Radiocarbon variations from tasmanian conifers: results from three holocene logs. Radiocarbon 37(2):361-9.

Buck C, Cavanagh W, Litton C. 1996. Bayesian approach to interpreting archaeological data. Chichester: Wiley.
Buck C, Christen J, James G. 1999. Bcal: an online bayesian radiocarbon calibration tool. Internet Archaeology 7. http://intarch.ac.uk.

Christen A, Nicholls G. 2000. Random-walk radiocarbon calibration. Technical report 457. Mathematics Department, Auckland University, New Zealand. http:// www.math.auckland.ac.nz/ nicholls.

Higham TFG, Hogg AG 1995. Radiocarbon dating of prehistoric shell from New Zealand and calculation of the $\delta$ r value using fish otoliths. Radiocarbon 37(2): 409-16.

Jones M, Nicholls GK. 1999. Datelab: new date analysis software. http://www.car.auckland.ac.nz. In prepara- 
tion.

McCormac FG, Hogg AC, Higham TFG, Baillie MGL, Palmer JG, Xiong L, Pilcher JR, Brown D, Hoper ST. 1998. Variations of radiocarbon in tree-rings: Southern Hemisphere offset preliminary results. Radiocarbon $40(3): 1153$.

McFadgen BG, Manning MR. 1990. Calibrating New Zealand radiocarbon dates of marine shells. Radiocarbon 32(2):229-32.

Nicholls GK, Jones MD. 1998. Radiocarbon dating with temporal order constraints. Technical report 407. Mathematics Department, Auckland University, New Zealand. http://www.math.auckland.ac.nz/ nicholls.

Ramsey CB. 1995. Radiocarbon calibration and analysis of stratigraphy: the OxCal program. Radiocarbon 37(2):425-30.

Sparks R, Melhuish W, McKee J, Ogden J, Palmer J, Molloy B. $1995 .{ }^{14} \mathrm{C}$ calibration in the southern hemi- sphere and the date of the last Taupo eruption: evidence from tree ring sequences. Radiocarbon 37(2): 155-63.

Stuiver M, Braziunas TF. 1993. Modeling atmospheric ${ }^{14} \mathrm{C}$ influences and ${ }^{14} \mathrm{C}$ ages of marine samples to 10,000 BC. Radiocarbon 35(1):137-91.

Stuiver M, Reimer PJ, Beck JW, Bard E, Burr GS, Hughen KA, Kromer B, McCormac FG, van der Plicht J, Spurk M. 1998. INTCAL98 radiocarbon age calibration, 24,000-0 cal BP. Radiocarbon 40(3):104183.

Vogel JC, Fuls A, Visser E, Becker B. 1986. Radiocarbon fluctuations during the third millennium BC. Radiocarbon 28(2A): $935-8$.

Vogel JC, Fuls A, Visser E, Becker B. 1993. Pretoria calibration curve for short lived samples, 1930-3350 BC. Radiocarbon 35(1):73-85. 